Ciencias de la Salud

\title{
Estado del esmalte dental después de retirar brackets y pulir el adhesivo residual a través de tres mecanismos, en premolares extraídos
}

\author{
State of the tooth enamel after removing brackets and polishing the residual \\ adhesive through three mechanisms, in extracted premolars. \\ Meibol Priscila Contero-Rosero, Ana Mishel Proaño-Rodríguez, \\ m_prisycr@hotmail.com, amproaño@uce.edu.ec
}

Universidad Central del Ecuador, Quito, Ecuador

Recibido: 26 de octubre de 2015

Aceptado: 2 de diciembre de 2015

\section{Resumen}

El presente trabajo tuvo como objetivo evaluar el daño producido al esmalte después de retirar brackets metálicos y pulir el adhesivo residual depositado sobre la superficie con tres tipos de instrumentos rotatorios. Un total de 45 premolares fueron extraídos por razones ortodónticas y divididos aleatoriamente en tres grupos $(\mathrm{n}=15)$ : Grupo A, piedra de Arkansas; grupo B, fresas multilaminadas (24 hojas) y grupo $\mathrm{C}$, fresas microdiamantadas. Cada uno de los especímenes fue examinado en el estereomicroscopio 0016813 en tres etapas: previo a la cementación de brackets, posterior a la remoción del bracket para definir la cantidad de adhesivo residual depositado sobre el esmalte a través del Índice de Adhesivo Residual (ARI); para esto se digitalizó cada imagen del adhesivo y se vectorizó en el programa de Autocad 2015 de Autodesk; finalmente, se evaluó el esmalte posterior pulido final con el fin de calificarlo según el Índice de Superficie del Esmalte (ESI). Se realizó un análisis comparativo utilizando el análisis de Lilliefors, lo cual indicó el uso de pruebas no paramétricas, Signo de Wilcoxon para comparación del estado inicial y final de cada grupo y Kruskal-Wallis para la comparación entre los grupos e índice de adhesivo residual. Diferencias estadísticamente significativas demostraron que las fresas multilaminadas ocasionaron menor daño manteniendo a los especímenes en un promedio de Índice de Superficie 2. 
Palabras clave: Daño del esmalte, adhesivo residual, piedra de Arkansas, fresas multilaminadas, fresas microdiamantadas.

\begin{abstract}
The aim of this research is to evaluate the damage produced to the dental enamel after the polish of the residual adhesive deposited on the surface, between three types of rotatory instruments. Methods: A total of 45 premolar were extracted, for orthodontic reasons, and divided randomly in three groups $(\mathrm{n}=15)$ : group $\mathrm{A}$, an Arkansas stone was used; group $\mathrm{B}$, multilaminated burs were used; group C, microdiamond burs were used. Each one of the samples was examined with the stereo microscope 0016813 in three phases: Before the cementation of the brackets, after the removal of the brackets, to define the amount of residual adhesive deposited on the enamel through the adhesive residual index (ARI) digitalizing each image of the adhesive and vectoring using the Autodesk program Autocad 2014, and finally, evaluating the enamel after the final polish with each of the instruments, with the purpose of classify them with the Enamel Surface Index (ESI) to perform the comparative analysis using Lilliefors Test through the non-parametric tests; Wilcoxon Sign to compare the initial and final state of each group, and the Kruskal-Wallis to compare between groups and adhesive residual index. Statistically significant differences made us conclude that multi-laminated burs caused less damage and maintained samples in an average Enamel Surface Index of 2.
\end{abstract}

Keywords: Enamel damage, residual adhesive, Arkansas stone, multilaminated bur, microdiamond bur.

\title{
Introducción
}

El esmalte dentario es el tejido más duro del organismo, ya que está constituido por millones de prismas altamente mineralizados que recorren toda su estructura, pero a pesar de ser el más duro, no se puede regenerar, por lo tanto, cualquier injuria que provoquemos, el daño será permanente (Zachrisson and Artun 1979).

En estudios acerca de la apariencia del esmalte posterior al tratamiento de ortodoncia se han observado que después de pulir el adhesivo residual, el esmalte presentaba alteraciones como: rayones, fisuras, fracturas y pérdida de su estructura, para lo cual se intentaba utilizar mecanismos de pulido mínimamente invasivos, pero no se conseguía un buen resultado, causando daños irreversibles al esmalte (Eliades et al. 2004). 
Las técnicas para retirar los brackets en forma segura se enfocan, principalmente, en fracturar la unión bracket-adhesivo, más que la unión adhesivo-esmalte; por consiguiente, la mayoría de los adhesivos quedan en la superficie de los dientes y deben ser pulidos con mucha delicadeza y cuidado del esmalte, para evitar la decoloración y retención de biopelícula por causa del adhesivo residual (Valleta et al. 2007).

Por lo tanto, es inevitable rayar el esmalte. El nivel de daño, alteración o deterioro se encuentra sujeto al instrumento utilizado; de esta manera, en los casos que se ha utilizado piedras de Arkansas el daño es muy evidente, aún más que en los que se ha empleado fresas de diamante (Herrera González 2014).

En la actualidad, muchos trabajos de investigación se han dedicado al estudio de posibles daños causados en el esmalte como resultado de la descementación de los brackets y del pulido final de esta estructura dentaria; sin embargo, no existe aún un consenso acerca de qué método de pulido final puede ser más seguro.

\section{Materiales y métodos}

La presente investigación fue realizada en 45 premolares extraídos en un consultorio privado por razones ortodónticas y recolectados con el consentimiento voluntario y autorización del paciente al que se explica que dichos premolares serán utilizados para este estudio. Los premolares fueron almacenados en suero fisiológico hasta la recolección total, experimental y de laboratorio, en donde se utilizaron distintas fresas de pulido e instrumentos rotatorios. Se necesitó un ambiente artificial controlado estrictamente. El presente estudio es comparativo, ya que evaluó qué tipo de pulido es menos agresivo para el esmalte dental en los distintos grupos de estudio (A, B y C) (Tabla 1); transversal, ya que las variables se analizaron en un solo momento y una vez terminado el estudio no hubo seguimiento ni proceso posterior.

Tabla 1. Distribución de la muestra de acuerdo con el tipo de pulido para la remoción del adhesivo residual posterior al descementado de brackets

\begin{tabular}{|l|l|}
\hline INSTRUMENTO UTILIZADO & $\mathbf{n .}^{\mathbf{0}}$ \\
\hline Piedra de Arkansas (Grupo A) & 15 \\
\hline Fresas multilaminadas 24 hojas (Grupo B) & 15 \\
\hline Fresas microdiamantadas (Grupo C) & 15 \\
\hline Total de la muestra & $\mathbf{4 5}$ \\
\hline
\end{tabular}


Se realizaron tres observaciones del esmalte de cada espécimen a través del estereomicroscopio aplicando el Índice de Superficie del Esmalte (ESI) para confirmar el estado idóneo de la muestra (Tabla 2).

Tabla 2. Índice del Estado del Esmalte (ESI)

\begin{tabular}{|l|l|}
\hline ESCALA & DESCRIPCIÓN \\
\hline 0 & Superficie perfecta, esmalte intacto, periquimatíes observables \\
\hline 1 & Superficie satisfactoria, esmalte con finas rayas, periquimatíes observables \\
\hline 2 & Superficie aceptable, esmalte con finas rayas, sin periquimatíes \\
\hline 3 & Superficie imperfecta, esmalte con rayas gruesas, sin periquimatíes \\
\hline 4 & Superficie inaceptable, con daño severo al esmalte y un rayado profundo \\
\hline
\end{tabular}

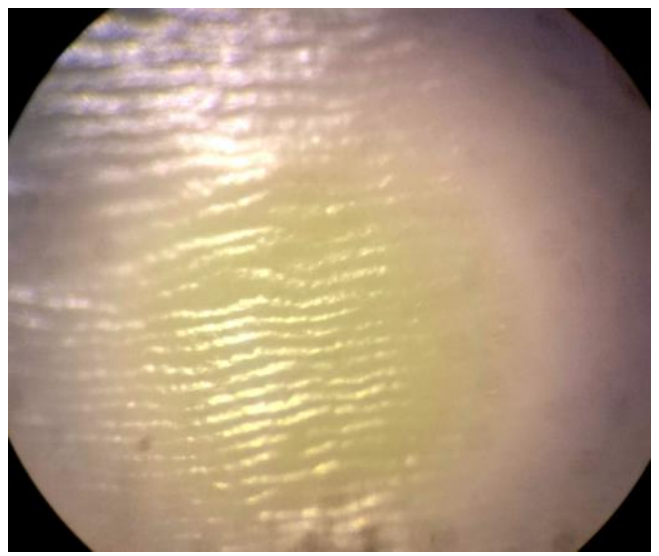

Figura 1. Primer registro fotográfico del esmalte previo al procedimiento

Se confeccionó una placa de acrílico donde fueron colocados uno por uno los premolares para mayor comodidad. Estos se almacenaron en suero fisiológico, el cual fue cambiado cada 2 días (Figura 2).
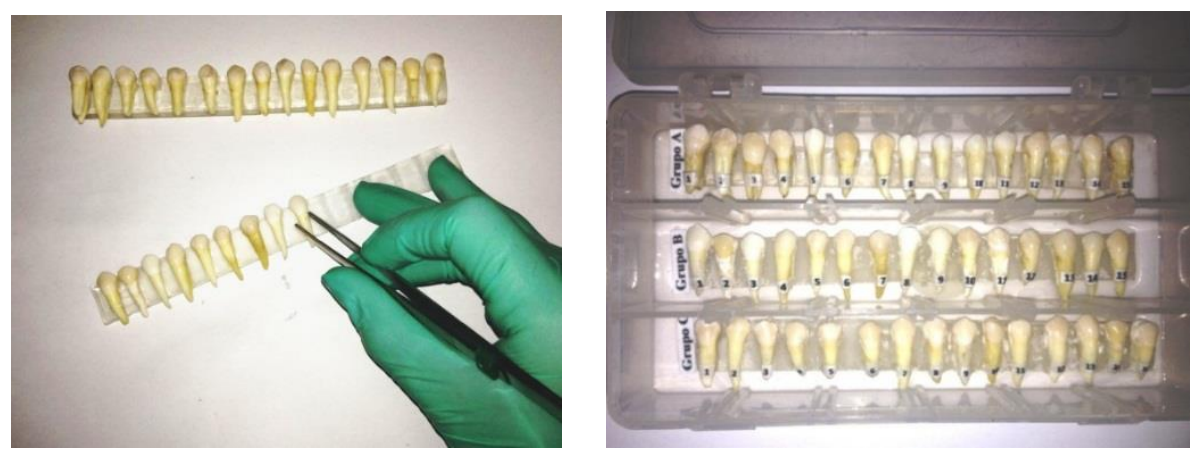

Figura 2. Colocación y numeración de la muestra en base de acrílico 
Los especímenes de cada uno de los grupos fueron limpiados con piedra pómez y agua destilada con la pieza de mano de baja velocidad. Posterior a esto fueron grabados con ácido fosfórico al $37 \%$ durante 15 s, según Graber, Vanarsdall y Vig (2013), y se procedió a lavarlos con agua durante $30 \mathrm{~s}$, luego se secaron minuciosamente.

Con un aplicador se realizó el sellado, es decir, la colocación del adhesivo de la misma marca comercial que la resina de cementación, formando una capa la cual se adelgazó con una ráfaga suave de aire para evitar cúmulo excesivo sobre el esmalte, como indica Herrera González (2014) (Figura 3).
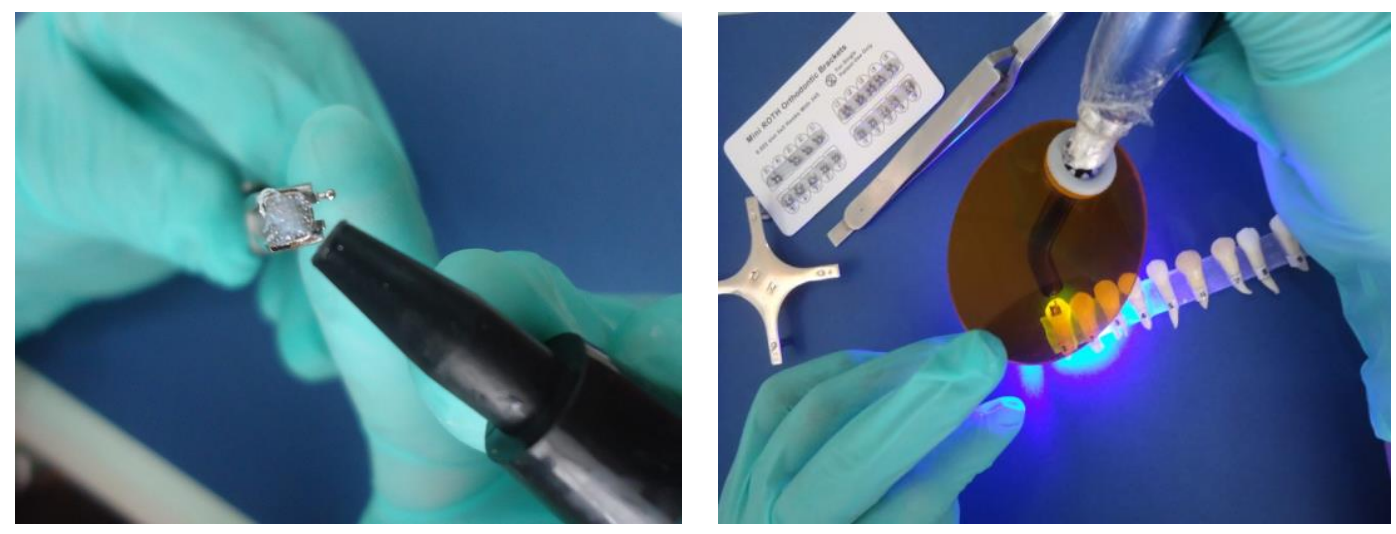

Figura 3. Preparación del bracket y fotopolimerización

Con un portabrackets se pinzó el bracket, se colocó la resina de cementación y se transportó a la superficie dental; se retiraron los excesos con un explorador, se fotopolimerizó con luz LED $(830 \mathrm{~nm})$ de acuerdo con el fabricante durante un total de $20 \mathrm{~s}: 10$ s por gingival y 10 por incisal. El almacenamiento de las unidades de estudio se realizó en un envase hermético durante $48 \mathrm{~h}$ para crear un ambiente artificial simulando el medio bucal, como menciona Rodríguez et al. (2013). Se determinó que el mejor medio es en suero fisiológico ya que esta disolución es compatible con microorganismos vivos.

La segunda observación se realizó después de la remoción del bracket con alicate sacabrackets, aplicando fuerzas tensiles y de torción y colocando los bocados en los bordes mesial-distal de la base del bracket (Figura 4). 


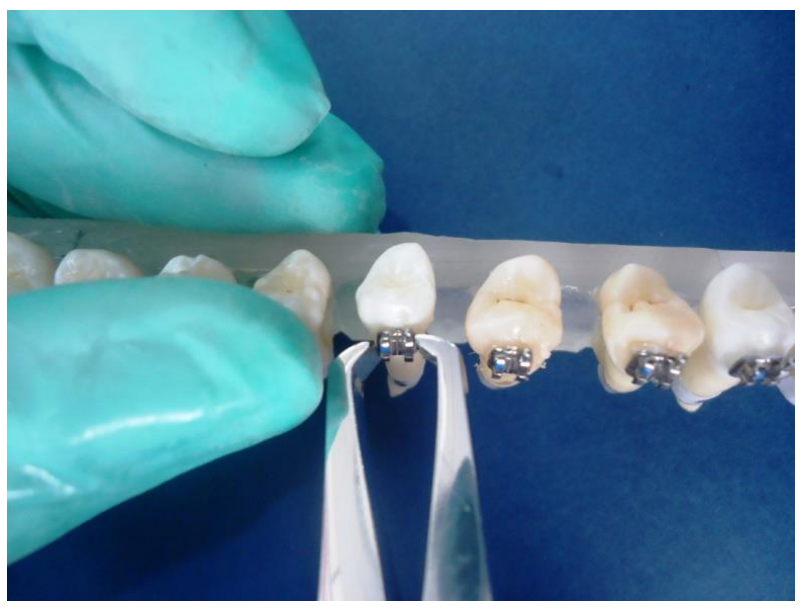

Figura 4. Descementación del bracket

Cada espécimen fue evaluado a través del estereomicroscopio para observar la cantidad del adhesivo residual depositado sobre la superficie del esmalte, para lo cual se realizó el segundo registro fotográfico. El mismo fue vectorizado en el programa Autocad 2015 (producto de Autodesk), con el objetivo de determinar el porcentaje de adhesivo y ubicarlo en la escala de Índice de Adhesivo Residual (ARI) (Tabla 3).

Tabla 3. Escala del Índice de Adhesivo Residual (ARI)

\begin{tabular}{|l|l|}
\hline Escala & Descripción \\
\hline 0 & Ausencia total del adhesivo residual en el diente \\
\hline 1 & Menos del $50 \%$ del adhesivo residual en el diente \\
\hline 2 & Más del $50 \%$ del adhesivo residual en el diente \\
\hline 3 & $100 \%$ del adhesivo residual en el diente con la impresión de la malla del bracket \\
\hline
\end{tabular}

Como tercer registro se observó la superficie del esmalte después de haber pulido el adhesivo residual de cada uno de los grupos de la siguiente manera:

Grupo A: las 15 unidades de estudio fueron sometidas al pulido con una piedra de Arkansas de forma cónica en un instrumental rotatorio de alta velocidad (Turbina NSK). Como indican Eminkahyagil et al. (2010), se realizaron movimientos próximo-proximales con una orientación tipo plano y refrigeración inicial. Después de haber pulido la mitad del adhesivo residual, se continuó con el procedimiento; pero sin refrigeración. Cada piedra se descartó después de pulir 2 especímenes. 
Grupo B: las 15 unidades de estudio fueron pulidas con fresas multilaminadas de 24 hojas, de forma cónica, de carburo tungsteno a alta velocidad (Turbina NSK) con refrigeración inicial y movimientos en sentido próximo-proximales. Antes de finalizar el pulido se retiró la refrigeración. La fresa se cambió cada 10 piezas en base al estudio realizado por Mejías Rotundo (2013).

Grupo C: los 15 especímenes fueron pulidos con una fresa microdiamantada de alta velocidad (Turbina NSK) con refrigeración, utilizando los mismos movimientos de las técnicas anteriores y con la refrigeración nula al finalizar el procedimiento. Según el estudio realizado por Moncada et al. (2009), la fresa se descartó después de ser utilizada en 5 piezas dentales.

\section{Resultados y discusión}

Los datos obtenidos tras las observaciones a través del estereomicroscopio y análisis del área de adhesivo residual en Autocad 2015 fueron procesados mediante una base de datos IBM SPSS (Stadistical Package for the Social Sciences) versión 21 (Figuras 5, 6 y 7).

Se identificó que ARI 1 (menos del 50\% de adhesivo) fue el más frecuente entre toda la muestra, ya que entre los grupos no hubo diferencia estadística significativa.
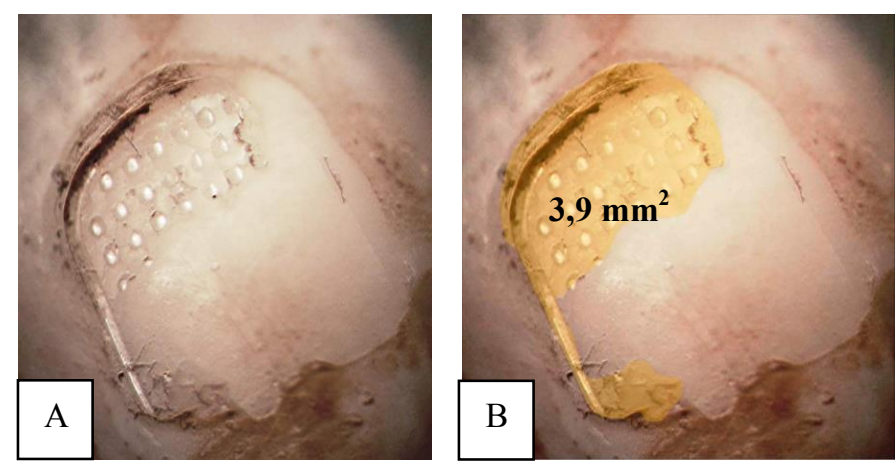

Figura 5. Índice de Adhesivo Residual en escala 1: A, fotografía tomada a través del estereomicroscopio; B, vectorización del área del adhesivo residual en Autocad 

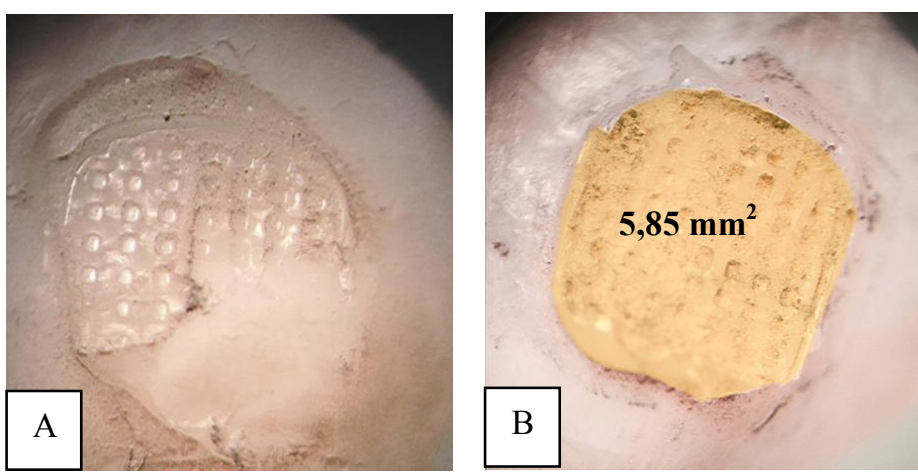

Figura 6. Índice de adhesivo residual en escala 2: A, fotografía tomada a través del estereomicroscopio; B, vectorización del área del adhesivo residual en Autocad
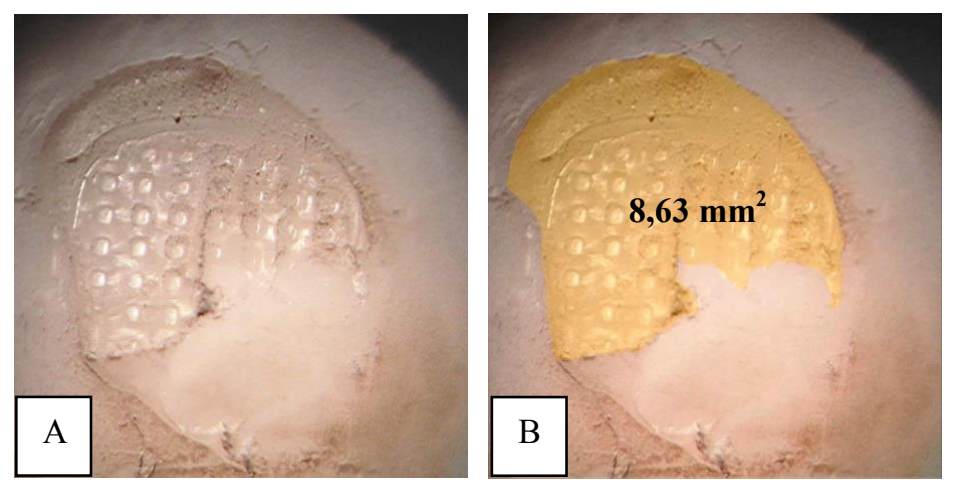

Figura 7. Índice de adhesivo residual en escala 3: A, fotografía tomada a través del estereomicroscópio; B, vectorización del área del adhesivo residual en Autocad

El pulido con piedra de Arkansas fue el que más daño causó al esmalte, dejándolo en un ESI 4, es decir, produjo una superficie inaceptable, con daño severo y rayado profundo.

La utilización de fresas multilaminadas fue exitosa, ya que después de pulir el adhesivo residual el esmalte tuvo un ESI 2, es decir, que aunque no se observaron periquimatíes, estas fresas brindaron una superficie aceptable con finas rayas. 
Tabla 4. Resultados del Índice de Superficie del Esmalte inicial y final de cada uno de los grupos

\begin{tabular}{|l|l|l|l|l|l|l|l|l|}
\hline GRUPO A & ESI-i & ESI-f & GRUPO B & ESI-i & ESI-f & GRUPO C & ESI-i & ESI-f \\
\hline A1 & 1 & 3 & B1 & 1 & 2 & C1 & 0 & 3 \\
\hline A2 & 1 & 4 & B2 & 1 & 2 & C2 & 0 & 3 \\
\hline A3 & 0 & 3 & B3 & 0 & 2 & C3 & 0 & 4 \\
\hline A4 & 0 & 4 & B4 & 1 & 2 & C4 & 1 & 2 \\
\hline A5 & 1 & 4 & B5 & 1 & 1 & C5 & 1 & 3 \\
\hline A6 & 1 & 4 & B6 & 1 & 2 & C6 & 1 & 3 \\
\hline A7 & 1 & 4 & B7 & 0 & 1 & C7 & 1 & 3 \\
\hline A8 & 1 & e & B8 & 1 & 1 & C8 & 0 & 2 \\
\hline A9 & 0 & 4 & B9 & 0 & 2 & C9 & 1 & 3 \\
\hline A10 & 1 & 4 & B10 & 0 & 1 & C10 & 1 & 3 \\
\hline A11 & 0 & 4 & B11 & 1 & 2 & C11 & 1 & 4 \\
\hline A12 & 1 & 4 & B12 & 1 & 2 & C12 & 1 & 2 \\
\hline A13 & 0 & 3 & B13 & 1 & 2 & C13 & 1 & 3 \\
\hline A14 & 0 & 3 & B14 & 1 & 2 & C14 & 1 & 4 \\
\hline A15 & 1 & 3 & B15 & 1 & 2 & C15 & 1 & 3 \\
\hline
\end{tabular}

Con las fresas microdiamantadas se obtuvo un promedio de ESI 3, es decir, causaron una superficie imperfecta con rayones gruesos en el esmalte. 

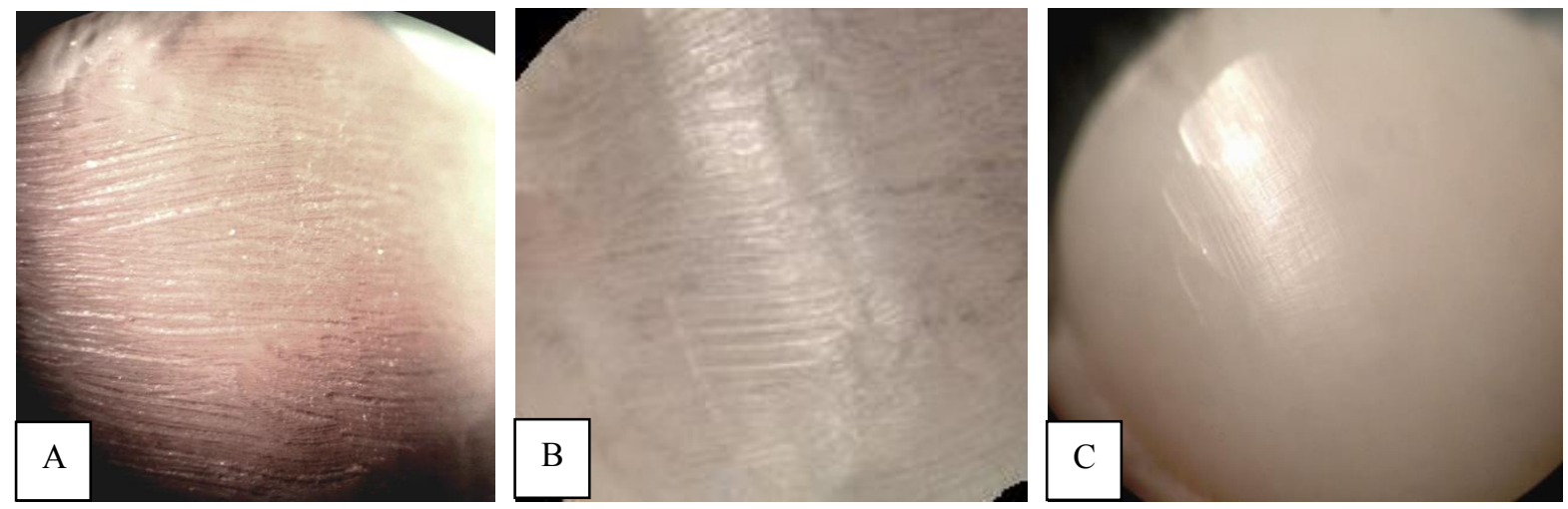

Figura 8. Vista en el estereomicroscopio posterior al pulido con: A, piedra de Arkansas; B, fresa microdiamantada; $\mathrm{C}$, fresa multilaminada

Eliades et al. (2004) obtuvieron que los mayores daños ocasionados al esmalte se realizaron con fresas de diamante y piedra de Arkansas, como resultado de su característica abrasiva. Este autor afirma que se deben usar solo en casos donde exista mayor cantidad de adhesivo residual, es decir, en un ARI 4 (el 100\% del adhesivo sobre el esmalte); sin embargo, señala que en la etapa final de remoción del adhesivo se debe cambiar de instrumento y utilizar fresas de corte como las multilaminadas, al contrario de las piezas analizadas en el presente estudio, en donde se puede observar escaso adhesivo y solamente se requiere un instrumento de corte, es decir, fresas multilaminadas, lo que ocasionará menos agresión y se obtendrá una superficie dentaria satisfactoria.

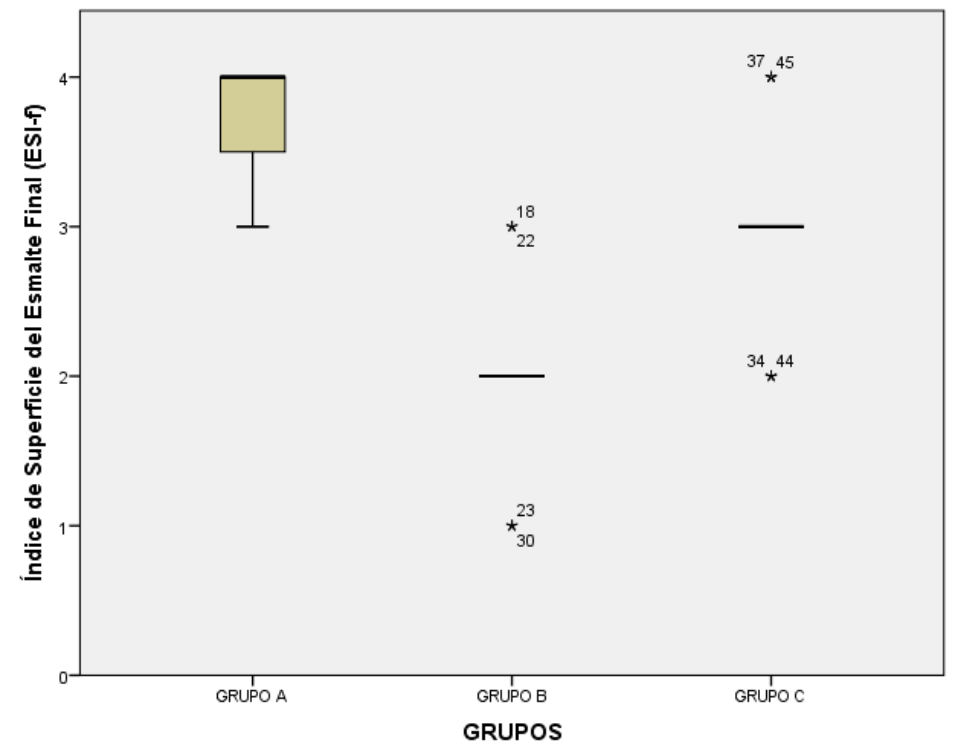

Figura 9. Diagrama de caja del Índice de Superficie del Esmalte final (ESI-f) de los grupos A, B y C 
Tabla 5. Rango promedio del ESI-f de los grupos A, B y C

\begin{tabular}{|l|l|l|l|}
\hline \multirow{2}{*}{$\begin{array}{l}\text { Índice de Superficie del Esmalte final } \\
\text { (ESI-f) }\end{array}$} & GRUPOS & N & Rango promedio \\
\cline { 2 - 5 } & GRUPO B & 15 & 34,63 \\
\cline { 2 - 5 } & GRUPO C & 15 & 24,00 \\
\cline { 2 - 5 } & Total & 45 & \\
\hline
\end{tabular}

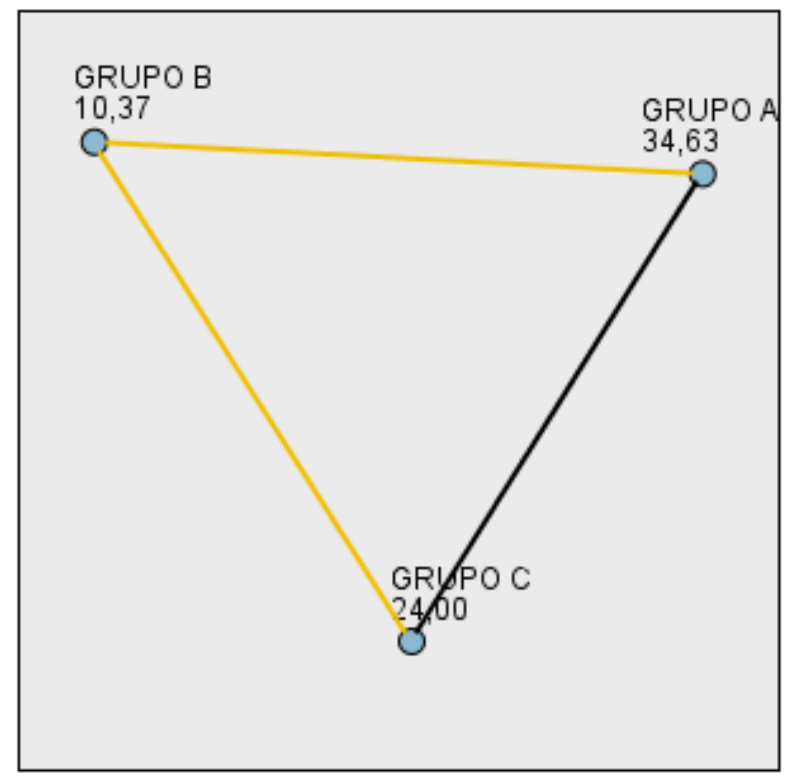

Figura 10. Comparación del ESI-f entre los grupos A, B y C

\section{Conclusiones}

El pulido con piedra de Arkansas fue el que más daño causó al esmalte, dejándolo en un ESI 4, es decir, produjo una superficie inaceptable, con daño severo y rayado profundo.

Con las fresas microdiamantadas se obtuvo un ESI 3, es decir, causaron una superficie imperfecta con rayones gruesos en el esmalte.

La utilización de fresas multilaminadas fue exitosa, ya que después de pulir el adhesivo residual

el esmalte tuvo un ESI 2, es decir, que aunque no se observaron periquimatíes, estas fresas brindaron una superficie aceptable con finas rayas. Por lo tanto, se determina que las fresas 
multilaminadas son las más indicadas para que el esmalte se encuentre en buenas condiciones después del tratamiento de ortodoncia.

\section{Referencias bibliográficas}

Campbell, P., 2010. Enamel Surfaces after orthodontic brackets debonding. Angle Orthodontic, $65,103$.

ELIADES, T. et al., 2004. Enamel surface roughness following debonding using two resin grinding methods. European Journal of Orthodontics, 26(3), 333-338.

EMINKAHYAGIL, N. et al., 2010. Effect of Resin-Removal Methods on Enamel and Shear Bond Strength of Rebonded Brackets. Angle Orthodontist, 76(2), 314-321.

Farzaneh A., Majid A., Javad A., (2013). Enamel Surface Roughness after debonding of orthodontic brackets and various Clean-up techniques. Journal of Dentistry. Vol 10: 1.

Gómez De Ferraris, M., Campos, A. (2003). Histología y Embriología Bucodental. Madrid: Médica Panamericana. (pp. 271-314).

GRABER, T.M.; VANARSDALL, R.L. y VIG, K.W.L., 2013. Ortodoncia: principios y técnicas actuales. 5ta ed. Madrid: Elsevier.

HERRERA GONZÁLEZ, G.R., 2013. Evaluación del esmalte dentario después de remover la resina residual posterior al descementado de brackets a través de dos tipos de sistemas. Especialidad. Universidad de Cuenca.

Maijer, R. (2005). Corrosion of orthodontic bracket bases. American Journal Orthodontics (56): p $43-48$

MEJÍAS ROTUNDO, O.J., 2013. Efecto de un sistema de pulido dental de óxido de aluminio de acuerdo al índice de superficie del esmalte. Revista latinoamericana de Ortodoncia y Odontopediatría. Disponible en: https://www.ortodoncia.ws/publicaciones/2013/art13.asp

Montasser, M., Drummond, J. (2009). Reliability of the Adhesive Remnant Index Score System with Different Magnifications. Angle Orthodontist, Vol 79. No. 4 p.773-776. 
Neslihan A. (2006). Shear Bond Stregth of Orthodontic brackets with 3 self - etch adhesives. AJODO; (129): 547-50.

Pramod K., Sinha, N. (2003). Aparatos ortodóncicos fijos de arco de canto y técnicas de adhesión. México: McGraw-Hill Interamericana Editores S.A.

Ravindra, N., Sunil. K, (2011). Terapias Actuales en Ortodoncia. Madrid: Elsevier

Scougall R., Zarate-Diaz C., Wakamatsu N., Nakagawa T. (2009). Resistencia al descementado de botones linguales colocados con diferentes sistemas adhesivos. Revista Espanola de Ortodoncia Vol. 39 No 1.pg 33.

Suárez, A. (2005). Nuevos brackets y aleaciones en ortodoncia: Ortodoncia Clínica y Terapéutica. (2da ed.). Barcelona: Masson. p. 341

Uribe, G. (2010). Ortodoncia: Teoría y Clínica. Medellin: Corporación para Investigaciones Biológicas.

VALLETA, R. et al., 2007. Evaluation of the debonding strenght of ortodontic brackets using three different bonding systems. Eur J Orthod. 29(6), 571-577.

ZACHRISSON, B.U. and ÅRTUN, J., 1979. Enamel surface appearance after various debonding techniques. Am J Orthodontic, 75(2), 121-127. 